\title{
Optical coherence tomography: guided primary percutaneous coronary intervention in acute myocardial infarction. A bridge too far?
}

\author{
Igor Kranjec ${ }^{1}$, Blaz Mrevlje², Jacek Legutko33, Dariusz Dudek ${ }^{3}$ \\ 'Department of Cardiology, Faculty of Medicine, University of Ljubljana, Ljubljana, Slovenia \\ 2Department of Cardiology, University Medical Centre Rostock, Rostock, Germany \\ ${ }^{3}$ Institute of Cardiology, Jagiellonian University Medical College, University Hospital, Krakow, Poland
}
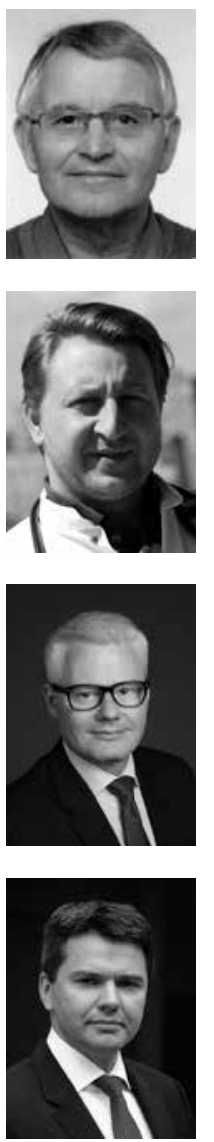

\section{RATIONALE}

The primary aim of percutaneous coronary intervention $(\mathrm{PCl})$ is to obtain excellent results in all attempted lesions without any procedure-related complications. However, it is equally important to guarantee that the benefits of revascularisation are durable. Stent implantation in the acute myocardial infarction (Ml) setting may allow various pathogenetic mechanisms to run aggressively, thus leading to further ischaemic events [1]. Ruptured vulnerable plaques containing large necrotic cores combined with increased platelet reactivity can create a potent pro-thrombotic environment. Massive thrombotic leftovers despite vigorous thrombo-aspiration as well as epicardial vasoconstriction, which is only partly responsive to nitrates, altogether give way to substantial underestimation of the true vessel size and, consequently, to the implantation of undersized stents.

Intravascular imaging certainly holds great potential for the discovery of most of the unfavourable morphologic features. Indeed, intravascular ultrasound studies (IVUS) have so far clearly demonstrated that stent under-expansion, alone or in combination with other PCI-related morphologies, contributed to the occurrence of stent thrombosis or restenosis [2]. Optical coherence tomography $(\mathrm{OCT})$ is a light-based imaging modality using near-infrared wavelength (range $\sim 1.3 \mu \mathrm{m}$ ). It shows superior axial resolution $(\sim 15 \mu \mathrm{m})$ compared with other intravascular systems currently used in vivo, such as IVUS $(\sim 150 \mu \mathrm{m})$ and angioscopy $(\sim 10-50 \mu \mathrm{m})$. Furthermore, the excellent resolution of OCT imaging makes it possible to visualise plaque characteristics only reported by histopathological studies in vivo [3].

Therefore, the aim of our review was to describe the current status of coronary intravascular OCT imaging with particular emphasis on acute MI patients. Has the prime time for routine OCT imaging to guide effectively primary $\mathrm{PCl}$ arrived yet?

\section{DECISION-MAKING DURING PCI}

Revascularisation strategy in patients with acute MI depends largely on the clinical presentation [4]. In ST-segment elevation $\mathrm{MI}$ (STEMI), the strategy is rather straightforward: reperfusion should be performed as early as possible, and primary PCI should be limited to the culprit vessel, with the exception of cardiogenic shock or persistent ischaemia. On the other hand, patients without ST-segment elevation MI (NSTEMI) represent a heterogeneous population. Those at (very) high risk should be considered for angiography within 2 to $24 \mathrm{~h}$. The revascularisation strategy should be based on the clinical status as well as the disease severity (distribution and angiographic lesion characteristics).

Numerous potential advantages and clinical applications of the OCT during elective as well as urgent $\mathrm{PCl}$ may be relevant in decision-making during $\mathrm{PCl}$ : pre-procedural evaluation of target coronary lesions and planning of the revascularisation technique, peri-procedural guidance of the $\mathrm{PCl}$ itself, and post-procedural assessment of $\mathrm{PCl}$-induced vascular injuries and their healing. Moreover, a lot of new exciting possibilities will keep our attention focused in the near future. In the setting of primary $\mathrm{PCl}$, however, the OCT imaging may be particularly helpful in the following four stages: (1) culprit lesion; (2) thrombus burden; (3) stent implantation; and (4) stent-related vascular injury and healing.

Address for correspondence:

Igor Kranjec, MD, PhD, FESC, Ass. Prof. of Medicine/Cardiology, Department of Cardiology, Faculty of Medicine, University of Ljubljana, Zaloska 7, 1000 Ljubljana, Slovenia, e-mail: igor.kranjec@gmail.com 


\section{ASSESSMENT OF CULPRIT LESION}

OCT characteristics for different components of atheromatous plaque have been validated in histology-controlled studies and confirmed in patients with various clinical presentations [3]. With regard to tissue characterisation, OCT allows us to identify three types of coronary plaque: fibrous, fibro-calcific, and lipid plaques [5]. Furthermore, OCT can distinguish several essential unstable features such as thin-cap fibroatheromas (TCFA), plaque ruptures, erosions, thrombi, and calcified nodules. Pathohistological features of a vulnerable plaque include a large lipid pool and a thin fibrous cap infiltrated with macrophages [6]. In the OCT images, necrotic lipid pools appear as diffusely bordered, signal-poor regions with overlying signal-rich bands corresponding to fibrous caps. TCFA is characterised by a large necrotic core (i.e. lipid present in $\geq$ two quadrants in any of the images within the plaque) with a thin fibrous cap $(<65 \mu \mathrm{m})$. Fibrous cap rupture is distinguished by the presence of fibrous cap discontinuity and a cavity formation of the plaque, while fibrous cap erosion is identified by the loss of the endothelial lining with lacerations of the superficial layers. Thrombus is recognised as a globular mass protruding into the vessel lumen from the surface of the vessel wall. Red thrombus appears as a high-backscattering structure with dorsal, signal-free shadowing, whereas white thrombus presents itself as a signal-rich, low-backscattering structure [5].

Acute $\mathrm{MI}$ is typically initiated by the sudden rupture or erosion of the vulnerable plaque followed by the thrombus formation and vascular spasm, altogether resulting in a critical luminal compromise. However, distinct pathological features and clinical presentations associated with the plaque ruptures, erosions, and calcified nodules suggest that they may be caused by different pathophysiologic mechanisms and, therefore, might deserve tailored treatment. It has been suggested that culprit lesions with lipid plaques, thinner fibrous caps, plaque ruptures $(>70 \%$ of all underlying mechanisms), fibrin-rich thrombi, and tighter obstructions are more common in patients with STEMI [7], whereas culprit lesions with fibrous or lipid plaques, thicker fibrous caps, plaque erosions ( $>60 \%$ of all underlying mechanisms), platelet-rich thrombi, and wider obstructions are more frequent in patients with NSTEMI [8]. The calcified nodules are the least common aetiology for acute $\mathrm{MI}$ ( $\sim 5 \%$ of all underlying mechanisms) and are more common in older patients [7]. A typical culprit lesion in the STEMI setting is shown in Figure 1.

OCT, performed as the pre-interventional imaging in acute MI patients, appears particularly useful: (a) when the culprit lesion is unclear (e.g. intermediate lesions), (b) in case of diagnostic uncertainties on coronary angiogram (e.g. haziness), and (c) to rule out significant atherosclerotic plaque encroachment when planning the "minimalist immediate mechanical intervention" [9].
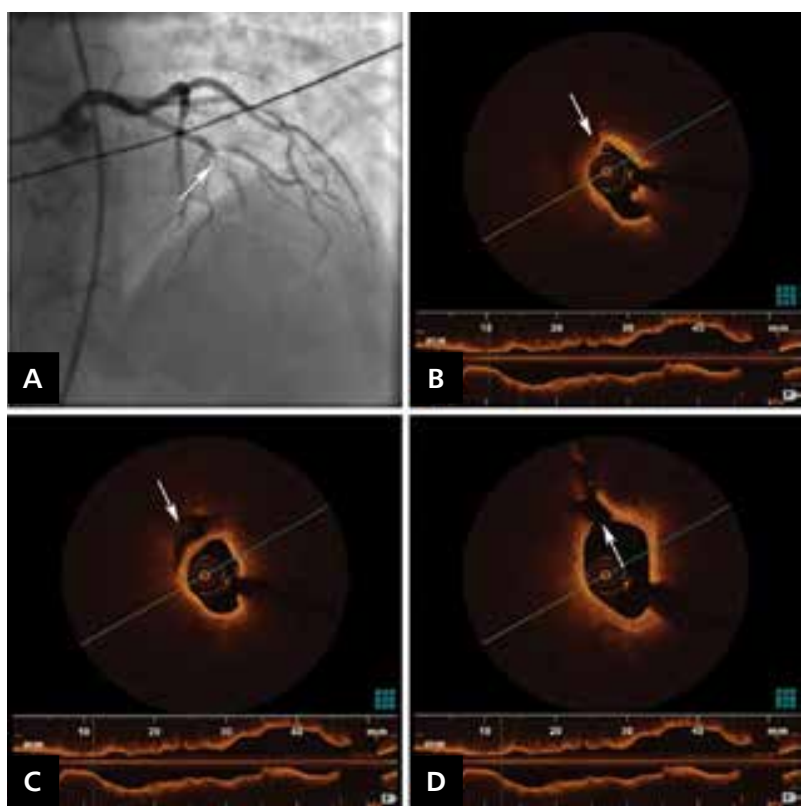

Figure 1. Culprit lesion in a 61-year-old man with an ST-segment elevation myocardial infarction; A. Angiography initially shows the mid-left anterior descending artery occlusion (arrow); B. Optical coherence tomography imaging after thrombus aspiration reveals a large lipid pool at the 11 o'clock position (arrow); C. Cavity formation is clearly visible at the same position (arrow) in front of the tightest vessel narrowing; D. The aperture of the ruptured plaque (arrow) is wide open against the direction of coronary blood flow

\section{ASSESSMENT OF THROMBUS BURDEN}

Large thrombi have been reported as independent predictors of post-procedural ischaemic events in patients treated with primary PCI for STEMI [10]. Despite mechanically achieving patent culprit arteries, up to $30 \%$ of these patients do not attain adequate myocardial tissue perfusion [11]. The reperfusion failure has been attributed to the no-reflow phenomenon related to distal embolisation of athero-thrombotic material, coronary vasoconstriction, and reperfusion injury. The complete removal of the culprit thrombus is carried out with the intention of avoiding, at least partly, the problem of inappropriate myocardial perfusion. The use of thrombectomy devices, particularly manual aspiration, has been shown to improve significantly the epicardial blood flow, myocardial blush grade, myocardial perfusion, and to decrease post-procedural ischaemic events [12]. However, some recent OCT studies have cast some doubts on the efficacy of the thrombectomy devices. In the study by Onuma et al. [11], manual thrombus aspiration failed to increase the coronary flow area as well as the stent area. Furthermore, OCT guidance for thrombus removal has lately been tested in two studies including $100 \mathrm{MI}$ patients. However, thrombus aspiration managed to remove only $\sim 50 \%$ of the intravascular thrombus burden, regardless of the initial thrombus amount, with similar rates for STEMI and 

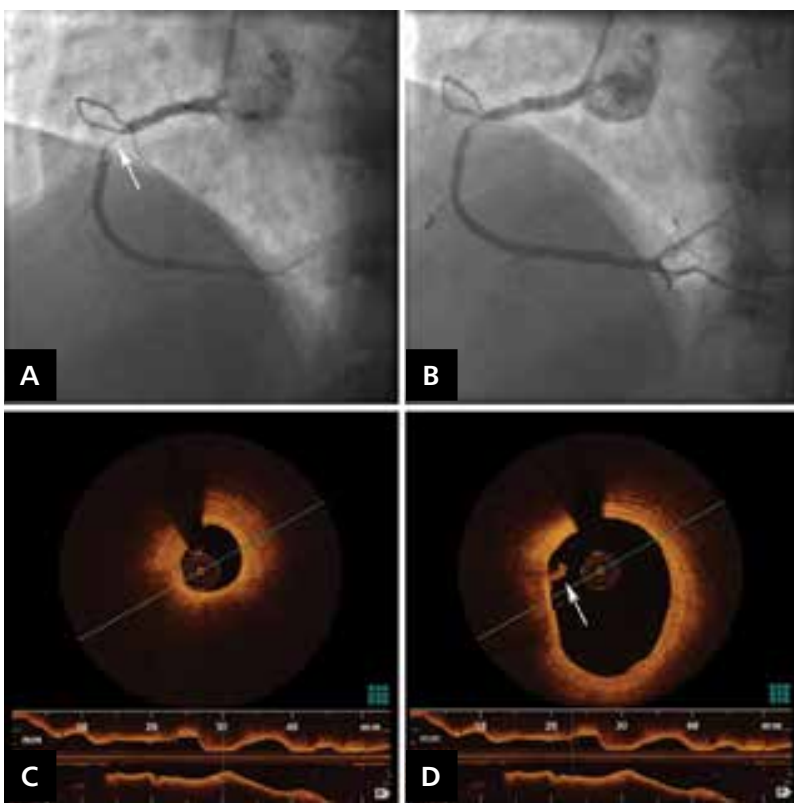

Figure 2. Successful thrombus aspiration in a 47-year-old gentleman with ST-segment elevation myocardial infarction; A. Angiography initially shows mid-right coronary artery sub-total occlusion with a large adjacent thrombus (arrow); B. Angiography after manual thrombus aspiration reveals a tight lesion devoid of any thrombus; C. Optical coherence tomography imaging after thrombus aspiration concurrently confirms a large fibro-lipid plaque; $\mathbf{D}$. Small thrombotic residues at the 9 o'clock position (arrow) indicate successful thrombus removal by means of manual thrombus aspiration

NSTEMI patients. Moreover, after stent deployment, OCT was always able to reveal some thrombotic masses despite the optimal angiographic result [13]. In another study, high-pressure dilatations performed even after extensive thrombo-aspiration led to an additional increase not only in stent but also in lumen areas. Fortunately, the measures of epicardial and tissue myocardial perfusion, as well as the clinical outcomes, were not detrimentally affected by such additional dilatations [14].

OCT has demonstrated excellent diagnostic potential to accurately detect intracoronary thrombi [7]. In the course of primary $\mathrm{PCl}$, OCT can be performed initially in incomplete coronary blockages to assess the overall thrombus burden and to guide selection of the most appropriate revascularisation strategy. However, the imaging should ideally be repeated after several thrombo-aspiration passages to check the residual thrombotic leftovers (Figs. 2, 3). In case of considerable thrombotic masses, the aspiration should be continued or more effective devices engaged.

\section{ASSESSMENT OF STENT IMPLANTATION}

It has already been mentioned that primary $\mathrm{PCI}$ is associated with significantly increased rates of post-procedural ischaemic events. Therefore, stent implantation should be optimal in

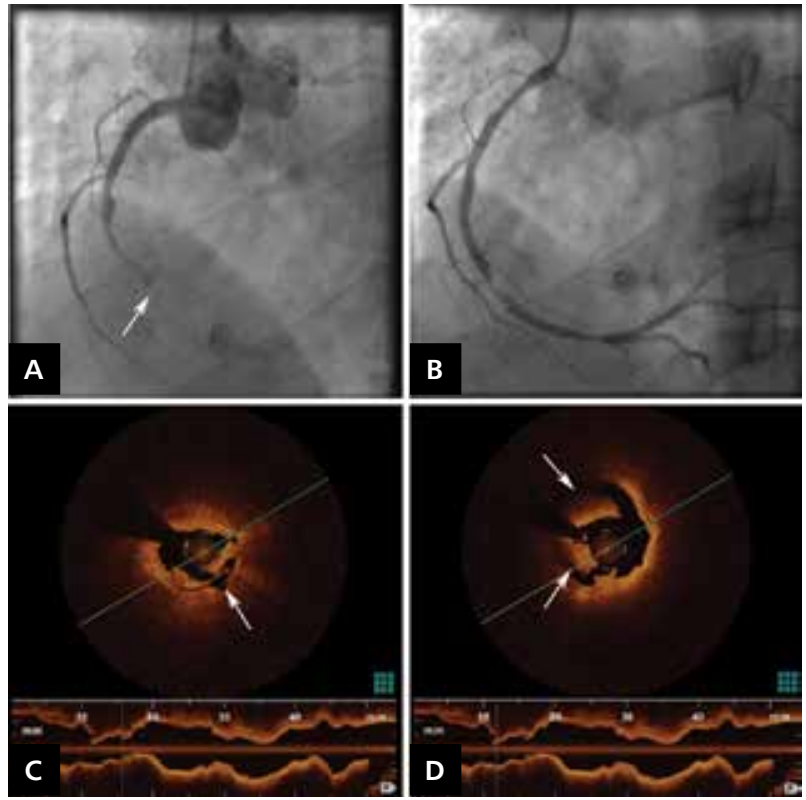

Figure 3. Unsuccessful thrombus aspiration in a 40-year-old man with ST-segment elevation myocardial infarction;

A. Angiography initially shows the mid-right coronary artery thrombotic occlusion (arrow); B. Angiography after manual thrombus aspiration reveals two tight lesions with possibly small thrombotic amounts; C. Optical coherence tomography imaging after thromboaspiration demonstrates a tight lesion with a large rupture at the 5 o'clock position (arrow); D. Large thrombotic masses distally at the 7-11 o'clock position (arrow) indicate unsuccessful thrombus removal by means of manual thrombus aspiration

the acute $\mathrm{Ml}$ setting to possibly improve short- and long-term clinical outcomes. OCT is thought to assist stent implantation by (a) finding appropriate landing zones for stent placement, (b) providing accurate measurements of target vessel width and tapering, lesion severity (particularly in NSTEMI) and length, and (c) evaluating stent expansion, apposition, and assessing residual stenosis after stent deployment.

Geometrical miss (GM) is an important indicator of suboptimal stent deployment. It is usually divided into two types, namely the longitudinal GM (i.e. diseased/injured vessel segment not fully covered by the stent) and the axial GM (i.e. balloon-artery size ratio $<0.9$ or $>1.3$ ). Mintz et al. [15] reported that only $6.8 \%$ of the angiographically normal reference segments were found to be truly normal on IVUS, while the average percentage of cross-sectional narrowing of the reference segments was $\sim 50 \%$. The recent STLLR trial (the Impact of Stent Deployment Techniques on Clinical Outcomes of Patients Treated With the CYPHER Stent) showed that GM occurred frequently in everyday $\mathrm{PCI}$ practice: overall GM in $66.5 \%$, longitudinal GM in $47.6 \%$, and axial GM in $35.2 \%$. Most of the difference in outcomes was due to the longitudinal GM [16]. Legutko et al. [17] demonstrated using 

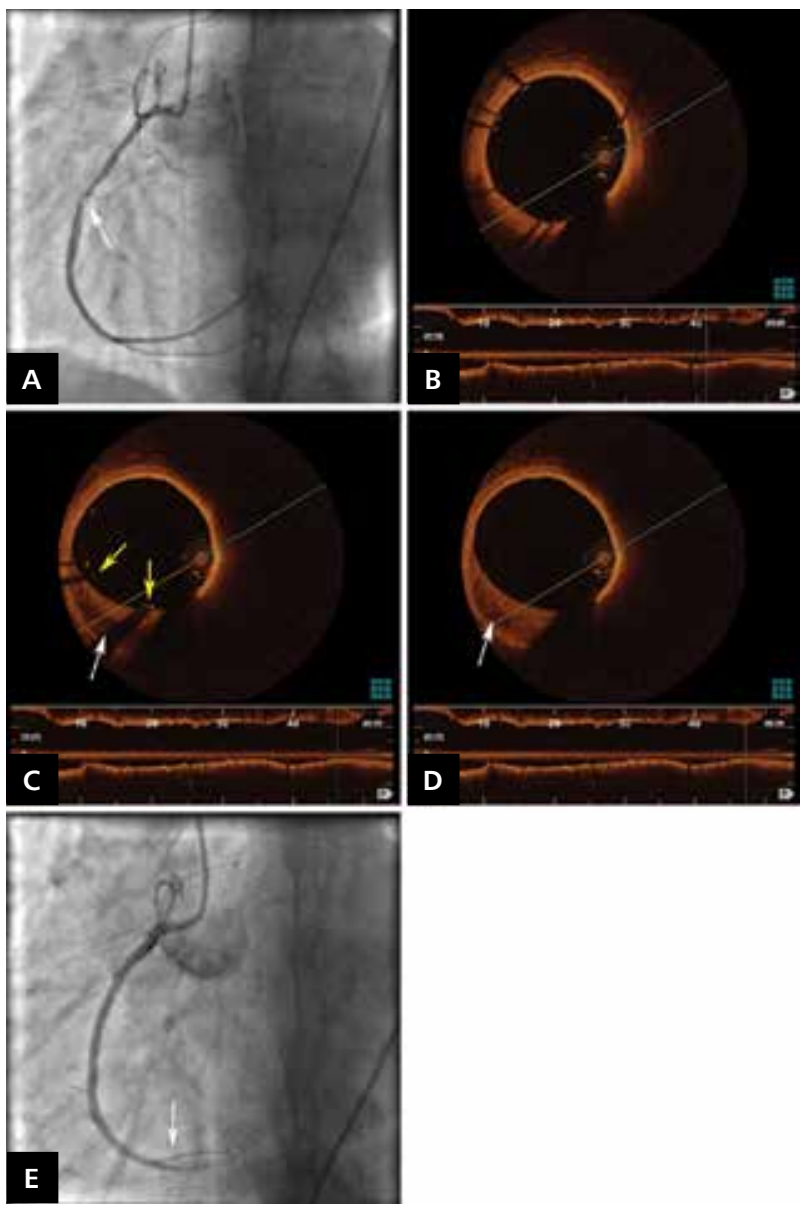

Figure 4. Inappropriate stent placement in a 47-year-old woman with ST-segment elevation myocardial infarction;

A. Angiography initially shows the mid-right coronary artery sub-total occlusion with a large adjacent thrombus (arrow);

B. Optical coherence tomography imaging after thrombus aspiration and stent implantation reveals mostly well apposed stent; $\mathbf{C}$. At the proximal stent edge, however, a few malapposed stent struts (yellow arrow) are placed on a large vulnerable plaque at the 5-9 o'clock position (white arrow); D. Vulnerable plaque (white arrow) largely protrudes beyond stent margins; E. Angiography after post-dilatation of the malapposed stent struts uncovers a no-reflow phenomenon (arrow)

virtual histology IVUS that the implanted stents failed to fully cover TCFAs in 35\% of NSTEMI patients and in 50\% of STEMI patients [18]. Incomplete coverage of the lipid-core lesions in unstable patients was likely associated with an increased risk for no-reflow, post-procedural (re)Ml, and plaque progression leading to edge restenosis [19]. To improve contemporary $\mathrm{PCl}$ practices, a stepwise approach targeting plaque burden at stent edges by means of OCT would be useful. A case of post-procedural no-reflow after the stent had been placed on a large lipid pool is shown in Figure 4.

Stent under-expansion is defined by (a) the in-stent minimal lumen area $\geq 90 \%$ of the average lumen area, and

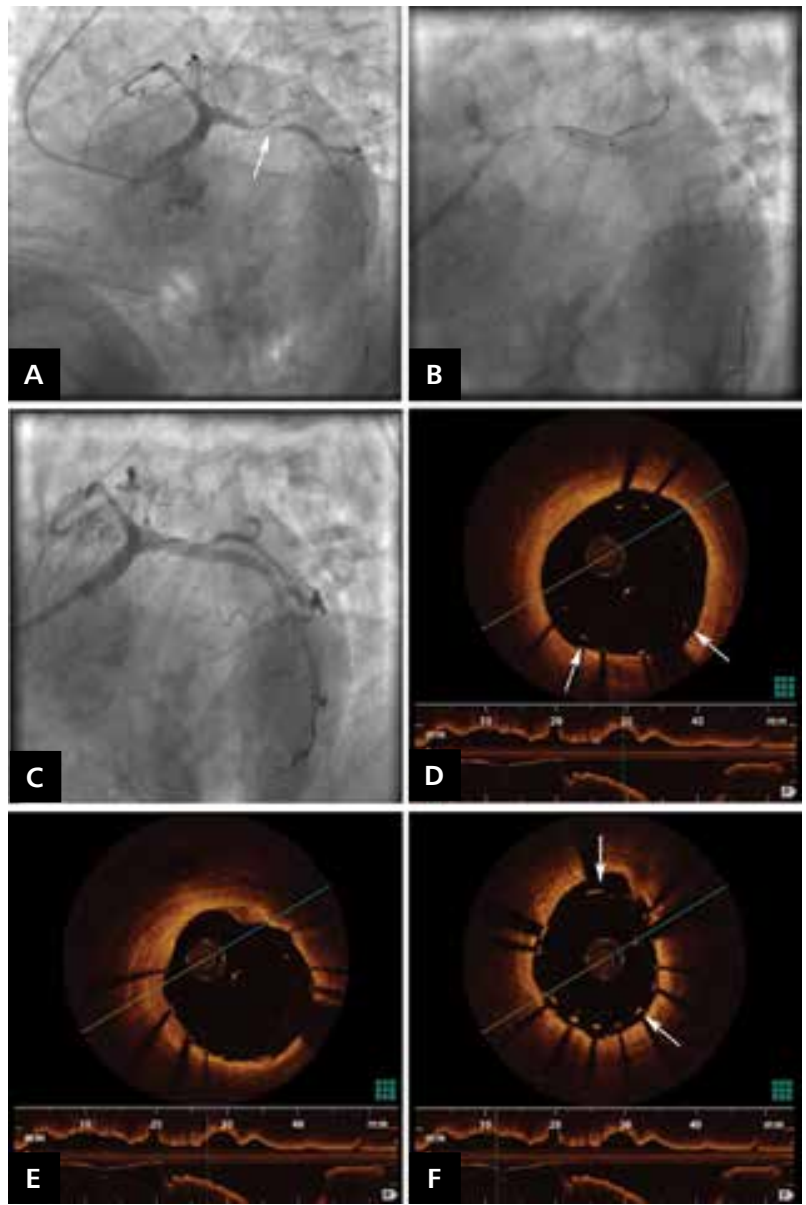

Figure 5. Insufficient stent apposition in a 70-year-old man with a non-ST-segment elevation myocardial infarction;

A. Angiography initially shows the proximal left circumflex artery sub-total occlusion (arrow); B. A drug-eluting stent $(3.0 \times 23 \mathrm{~mm})$ is deployed at 16 atmospheres; C. Angiography after stent implantation reveals an acceptable result without any significant residual stenosis; D. Optical coherence tomography imaging demonstrates a substantial malapposition of the proximal stent struts at the 6-14 o'clock position (arrow); E. Stent struts are well apposed only in a short mid-stent segment; F. Distinct malapposition of stent struts is seen distally at the 3-12 o'clock position (arrow)

(b) $\geq 100 \%$ of lumen area of the reference segment with the lowest lumen area. Incomplete stent apposition occurs if there is a visible separation of a stent strut from the vessel wall (sum of strut thickness + abluminal polymer thickness). Finally, reference luminal narrowing is defined as a lumen area $<4.0 \mathrm{~mm}^{2}$ [20]. In a mixed population containing $41 \%$ of patients with stable coronary artery disease and $60 \%$ with acute coronary syndrome, Prati et al. [21] recognised stent under-expansion in $11.4 \%$ and incomplete stent apposition in $29.7 \%$. Significant reference lumen narrowing was, somewhat surprisingly, only reported in $2.8 \%$ [20]. A case of incomplete stent apposition in a patient with acute $\mathrm{MI}$ is shown in Figure 5. 


\section{ASSESSMENT OF STENT-RELATED VASCULAR INJURY AND HEALING}

Primary $\mathrm{PCl}$ is the approved reperfusion therapy in STEMI as well as in high-risk non-STEMI patients and stenting is recommended over balloon angioplasty alone [4]. Yet the PCI itself is inevitably associated with an extensive vascular injury because the improvement in lumen dimensions is a result of vessel stretch, plaque compression, medial dissection, and embolisation of plaque constituents. Early after stenting, fibrin, platelets, and acute inflammatory cells are nearly always observed in association with stent struts. However, the concept that stents provide a boundary that excludes the underlying plaque from the lumen is not supported by pathomorphological studies because penetration of stent struts into a lipid core is quite common [1]. It is reasonable to expect that high-resolution imaging such as OCT will reveal a lot of intraluminal abnormalities in patients undergoing primary $\mathrm{PCl}$.

In the OCT studies, acute effects of stent implantation on the vessel wall have been described as tissue prolapse, intra-stent dissection, edge dissection, and intra-stent thrombi [20]. Tissue prolapse is a convex-shaped protrusion of tissue between adjacent stent struts toward the lumen, without disruption of the continuity of the luminal vessel surface. The distance from the arc connecting adjacent stent struts to the greatest extent protrusion should be $>50 \mu \mathrm{m}$. Intra-stent dissection is a disruption of the luminal surface in the stent segment. It can appear in two forms, as a linear rim of tissue with a clear separation from the vessel wall, plaque, or stent struts, or as an empty cavity behind the struts. Conversely, edge dissection is defined as the presence of a linear rim of tissue with a width of $\geq 200 \mu \mathrm{m}$ and a clear separation from the vessel wall of plaque that is adjacent $(<5 \mathrm{~mm})$ to a stent edge. Gonzalo et al. [22] investigated 73 consecutive patients, 41 with stable coronary artery disease and 32 with acute coronary syndrome. They reported tissue prolapse in $97.5 \%$, intra-stent dissection in $91.1 \%$, and edge dissection in $21.4 \%$. The rate of different features of vascular damage was similar in diverse clinical presentations although the tissue prolapse was found to be more extensive in unstable patients. Typical OCT features of stent-related vascular injuries in acute MI patients are shown in Figures 6-8.

Some of the post-procedural OCT findings appear disturbing at first glance, although they are angiographically invisible. They may trigger unnecessary additional interventions. Therefore, further refinement of the decision-making process during $\mathrm{PCl}$ is warranted. In general, acute findings after stenting, such as edge dissections, tissue protrusions, and incomplete stent apposition, detectable only on OCT, tend to be smaller at the follow-up than those seen on both OCT and IVUS. Most of them will resolve completely. Likewise, serial OCT data strongly suggest that the rates of incomplete stent apposition spontaneously decrease through the filling of the stent-lumen gap with neointima, without any clinical
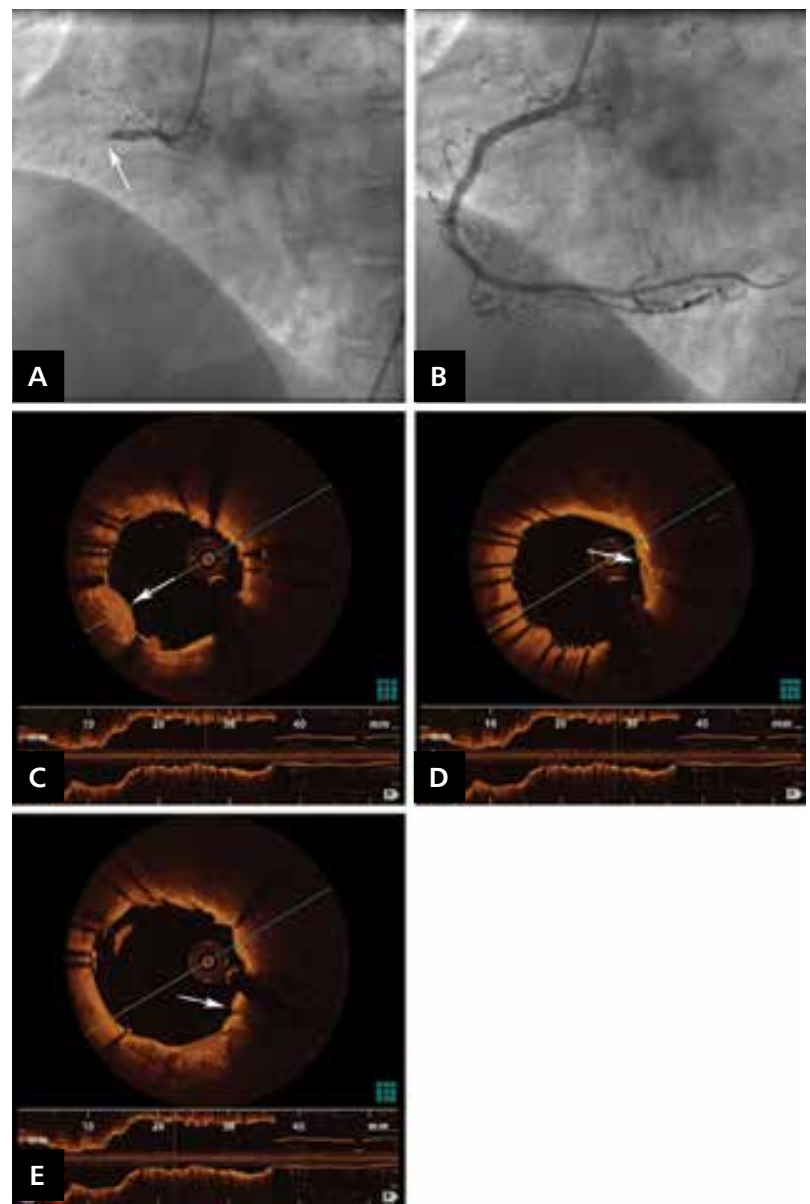

Figure 6. Multiple optical coherence tomography abnormalities in an 80-year-old woman with a non-ST-segment elevation myocardial infarction; A. Angiography initially shows proximal right coronary artery occlusion (arrow); B. Angiography after stent implantation shows a satisfactory result. Optical coherence tomography imaging, however, reveals numerous abnormal features, such as tissue prolapse (C, arrow), intra-stent dissection (D, arrow), and intraluminal thrombus ( $\mathbf{E}$, arrow)

events at mid-term follow-up. Gutierrez-Chico et al. [23] investigated in more detail the relationship between various limits for incomplete stent apposition and whether or not it persisted throughout the 12-month follow-up. They realised that strut-lumen distances $<270 \mu \mathrm{m}$ resolved in all cases and those $<400 \mu \mathrm{m}$ resolved in $93 \%$ of cases, without any occurrence of ST.

Previous reports have suggested an association between angiographic as well as IVUS-detected edge dissections and early ST. These and other studies consistently showed that, in addition to edge dissections, other abnormal findings were concurrently present, most consistently a low minimal lumen diameter/area, poor stent expansion, and a reduced final epicardial flow $(\mathrm{TIMI}<3)$. Although there is not yet sufficient data to indicate which angiographically silent edge dissections will lead to adverse events, and thus require ad- 

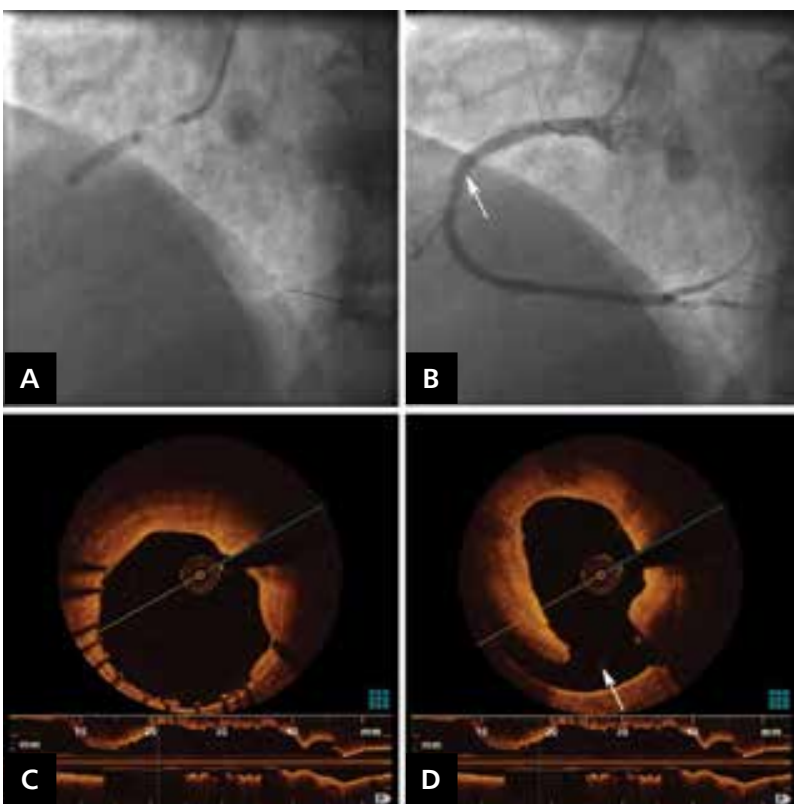

Figure 7. Angiographically visible edge dissection in a 47-year-old man with ST-segment elevation myocardial infarction; A. Angiography after stent implantation shows dissection at the distal stent edge; $\mathbf{B}$. Stent placement at the mid-right coronary artery segment (arrow); C. Stent struts at the distal stent edge are acceptably well apposed; $\mathbf{D}$. Large dissection beyond the distal stent edge is seen at the 6 o'clock position (arrow)
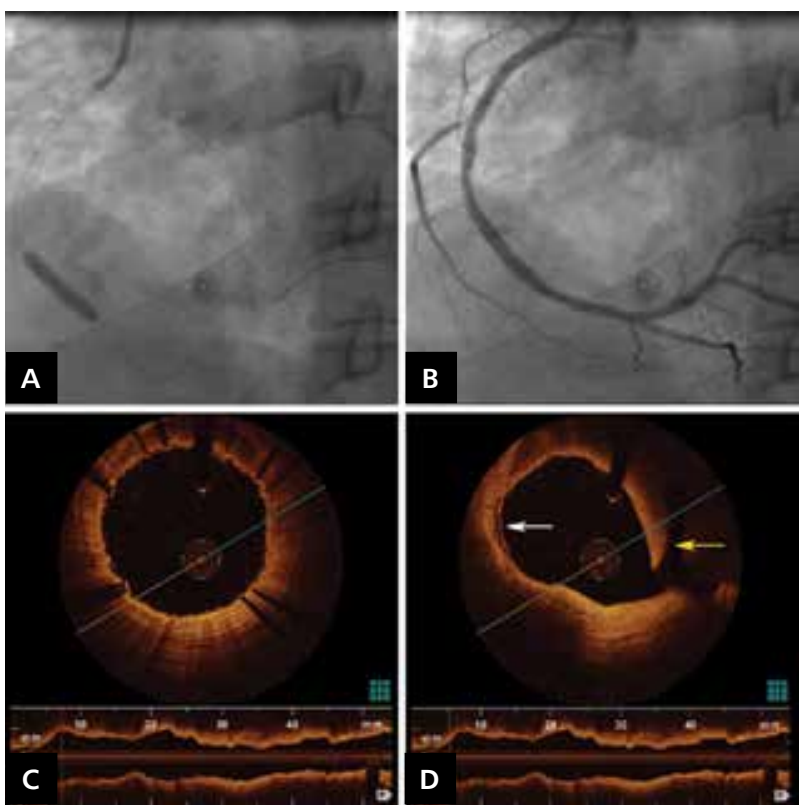

Figure 8. Angiographically silent edge dissection in a 40-year-old man with an ST-segment elevation myocardial infarction; A. Angiography after stent implantation does not reveal any major dissections; B. Stent positioning at the mid-right coronary artery segment; $\mathbf{C}$. Stent struts at the distal stent edge are well apposed; D. Large dissection beyond the distal stent edge is seen at the 3 o'clock (yellow arrow) and 9 o'clock (white arrow) positions
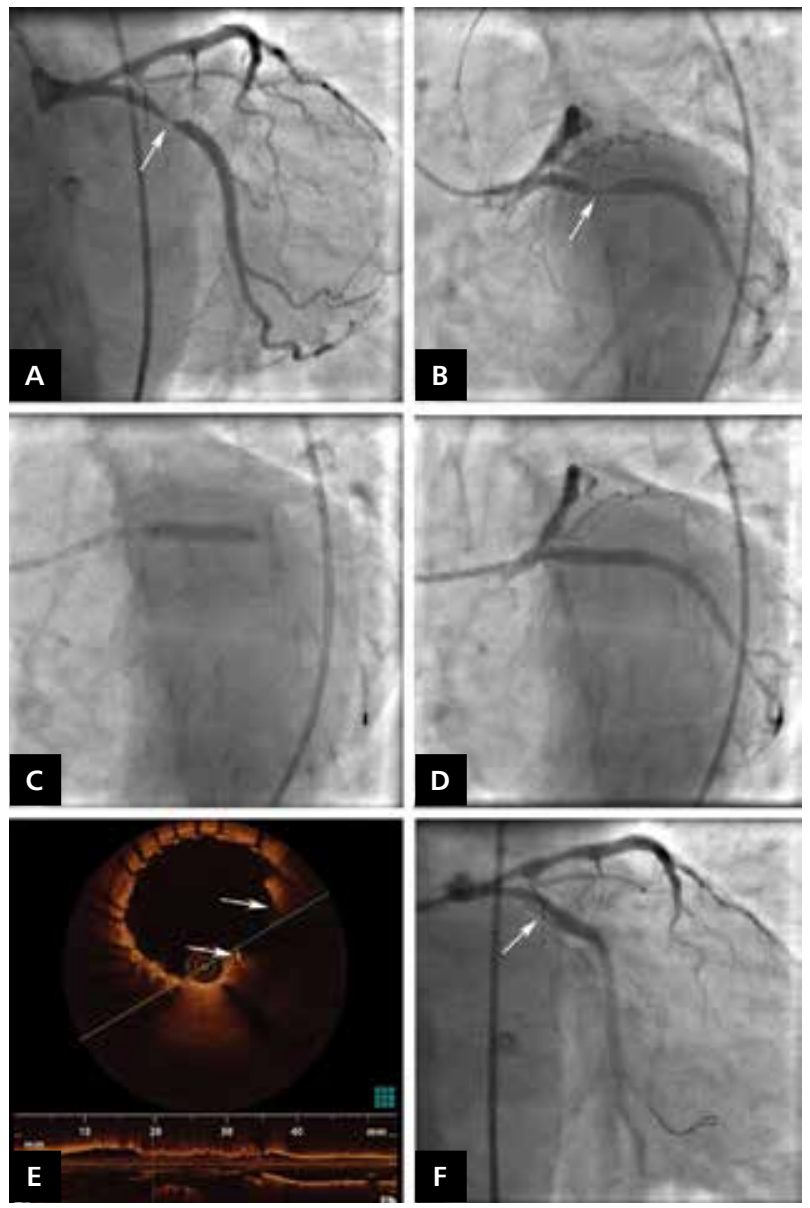

Figure 9. Vessel dissection resulting from over-aggressive dilatation in a 63-year-old woman with a non-ST-segment elevation myocardial infarction; A. Tight proximal left circumflex artery stenosis in anteroposterior caudal view (arrow); B. Same lesion in left anterior oblique (LAO) caudal view (arrow); C. Implantation of bare metal stent $(4.5 \times 18 \mathrm{~mm})$ at 18 atmospheres in LAO caudal view; D. Angiography after stent implantation in LAO caudal view shows a "waist" in the middle of the stent;

E. Optical coherence tomography imaging after stent implantation reveals insufficient stent expansion with intraluminal tissue prolapse at the 2-7 o'clock (arrow) position; F. High-pressure post-dilatation with too large balloon resulted in a Type $C$ dissection (arrow) that needed implantation of an additional stent

ditional intervention, it is thought-provoking that only major abnormalities, such as longitudinal extensions $>8 \mathrm{~mm}$ by IVUS and circumferential extensions $>3 \mathrm{~mm}$ by OCT, will be associated with an adverse long-term clinical course [24].

\section{PROCEDURAL AND CLINICAL CONSEQUENCES OF OCT IMAGING}

Despite the emerging use of OCT in everyday interventional practice, prospective randomised clinical trials testing the ability of OCT to guide elective or primary $\mathrm{PCI}$ have not been performed so far. Nevertheless, CLI-OPCI study (the Centro per la 
Lotta contro I'Infarto-Optimisation of Percutaneous Coronary Intervention) analysed a total of 670 patients: 264 patients with stable angina, 197 patients with NSTEMI, and 209 patients with STEMI [21]. 335 patients underwent OCT-guided $\mathrm{PCl}$ (study group) and 335 patients were managed with angiographic guidance alone (control group). The following actions were recommended when OCT disclosed significant procedural issues defined by protocol: (1) edge dissection and reference lumen narrowing required the implantation of an additional stent at the edge of the previously implanted stent; (2) stent underexpansion required further dilation of the previously implanted stent with a non-compliant balloon of the same diameter at $\geq 18$ atmospheres (atm) or with a semi-compliant balloon having a diameter $\geq 0.25 \mathrm{~mm}$ larger than the previously used balloon at $\geq 14 \mathrm{~atm}$; (3) incomplete stent apposition required further dilation of the previously implanted stent with a non-compliant or semi-compliant balloon having a diameter $\geq 0.25 \mathrm{~mm}$ larger than the previously used balloon at $\geq 14$ atm; and finally (4) thrombus required further dilation of the previously implanted stent with a non-compliant or semi-compliant balloon of the same diameter at 8-14 atm for $60 \mathrm{~s}$. Additional interventions according to the adverse intracoronary OCT findings were needed in as many as 34.7\% of the patients. Specifically, further dilatation was performed in $22.1 \%$ of cases: in $14.0 \%$ to fix stent under-expansion and in $8.1 \%$ to reduce intra-stent thrombus. Additional stenting was performed in $12.6 \%$ of cases.

Short-term outcomes were similar in the OCT and control groups: cardiac death occurred in $0.6 \%$ in the study group vs. $0.9 \%$ in the control group $(p=0.1)$, whereas $\mathrm{Ml}$ occurred in $3.9 \%$ in the study group vs. $6.5 \%$ in the control group ( $p=0.118$ ). On the other hand, unadjusted analysis at mid-term follow-up showed that the OCT group had a significantly lower one-year risk of cardiac death (1.2\% vs. $4.5 \%$ in the control group, $\mathrm{p}=0.010)$, cardiac death or $\mathrm{MI}(6.6 \%$ vs. $13.0 \%$ in the control group, $p=0.0006)$, and a composite of cardiac death, $\mathrm{MI}$, or repeat revascularisation $(9.6 \%$ vs. $14.8 \%$ in the control group, $p=0.044)$. OCT guidance was associated with a significantly lower risk of cardiac death or $\mathrm{Ml}$ even at extensive multivariable analysis adjusting for baseline and procedural differences between the groups and at propensity-score adjusted analyses. Unfortunately, potential differences in clinical outcomes between stable and unstable patients were not reported.

\section{FEASIBILITY, SAFETY, AND EFFICACY OF OCT IMAGING}

Current European Society of Cardiology guidelines on myocardial revascularisation, for the first time, recommend OCT as a tool for assessment of mechanisms of stent failure (class II a, level C) and in selected patients to optimise stent implantation (class II b, level C) [4]. The feasibility and safety of the endovascular imaging in critically ill patients, however, depend on the mechanical characteristics of the imaging probes, the additional time required for the set-up and data acquisition, and the necessity to provisionally occlude the investigated vessel. Early commercially available OCT versions used time domain detection that required proximal occlusion of the investigated coronary artery. Currently available systems, instead, utilise frequency domain detection and do not require vessel occlusion. New OCT probes are low profile (2.6 French), flexible, with hydrophilic coating, and the acquisition speed is at least ten times higher compared with IVUS. The examined artery is rendered free of blood by means of iso-osmolar X-ray contrast injected by power injector at rates 2 to $4 \mathrm{~mL} / \mathrm{s}$ (total volume $\sim 30 \mathrm{~mL}$ ), whereas low molecular weight dextran is widely used. Automated 20 to $40 \mathrm{~mm} / \mathrm{s}$ pullback allows for imaging of a 54 to $75 \mathrm{~mm}$ coronary segment during a 3-s injection. New high-resolution systems also provide for three-dimensional reconstruction with a $360^{\circ}$ panoramic view of the vessel. The radial access tolerates OCT imaging though a trend towards less optimal image quality and more artifacts have been observed. Particular care should be taken in patients with renal insufficiency, haemodynamic instability, severely impaired contractility, last remaining coronary vessel, and allergy to flush medium. The imaging may be less successful in vessels with extreme tortuosity, large vessels ( $>4.5 \mathrm{~mm})$, very small vessels $(<1.5 \mathrm{~mm})$, and poor fitting guide catheter. The vessel situation can be significantly worsened with occlusive OCT or guiding catheter, and with extensive vessel dissections. To date, limited experience does not suggest an increased risk in thrombosed lesions. Imaging of the left stem and bifurcations is undertaken within the limits of the penetration depth.

The old, occlusive time domain system sometimes caused ST-segment (re)elevations, although they were irreversible. On the other hand, imaging with the newer frequency domain system was not associated with any major complications because no cases of significant arterial spasm, dissection or life-threatening arrhythmias (i.e. requiring pharmacologic therapy, revascularisation, or cardioversion/defibrillation) were reported [21]. Furthermore, no significant differences in post-procedural renal function were found in the OCT group as compared with the control group. However, some level of common sense to interpret the imaging findings is advised because too much aggressiveness may cause unnecessary vessel damage (Fig. 9).

Cost-effectiveness studies for the OCT-guided primary $\mathrm{PCl}$ in acute $\mathrm{MI}$ have not been performed yet. However, some deductions can possibly be made from similar IVUS studies. Mueller et al. [25] found that in-hospital costs for procedural personnel, capital equipment, and disposable devices were evidently higher in the IVUS group compared with the angiographically guided controls. On the other hand, two-year major adverse event-free survival was significantly higher in the IVUS-guided group (80\% vs. $69 \%$ in angiographically-guided 
group, $p<0.040)$. The authors concluded that, when used in a provisional stenting strategy, routine IVUS imaging is cost-efficient half of the time. Considering the unrivalled high resolution of the OCT imaging combined with its ease of use, the prospect of OCT becoming an indispensable imaging tool even in high-risk acute $\mathrm{MI}$ patients is challenging.

\section{CONCLUSIONS}

OCT imaging with its high resolution and ease of use seems promising in decision making during primary $\mathrm{PCl}$, including evaluating controversial culprit lesions, assessing thrombus removal, facilitating stent implantation, and surveying stent-related vascular injury. When thoroughly tested in properly designed and conducted clinical trials, the imaging will certainly become a standard companion in the setting of primary $\mathrm{PCl}$ in the acute $\mathrm{MI}$ setting and complex $\mathrm{PCl}$. All being well, our combined endeavours will assist us to reduce the unacceptably frequent post-procedural ischaemic events after the primary $\mathrm{PCl}$.

\section{Acknowledgements}

We are grateful to our colleagues (Dr M. Bunc, Dr M. Noč, Dr M. Čerček) who were willing to share their cases and achievements with us. We also acknowledge the generous help of other colleagues (Dr J. Jakala, Dr P. Kleczynski, Dr L. Rzeszutko, Dr C.A. Nienaber) in writing this paper.

Conflict of interest: none declared

\section{References}

1. Nakazawa G, Finn AV, Joner M et al. Delayed arterial healing and increased late stent thrombosis at culprit sites after drug-eluting stent placement for acute myocardial infarction patients. An Autopsy study. Circulation, 2008; 118: 1138-1145.

2. Sonoda S, Morino Y, Ako J et al., for the SIRIUS Investigators. Impact of Final Stent Dimensions on Long-Term Results Following Sirolimus-Eluting Stent Implantation Serial Intravascular Ultrasound Analysis From the SIRIUS Trial. J Am Coll Cardiol, 2004; 43: 1959-1963.

3. Jang IK, Tearney GJ, MacNeil B et al. In vivo characterization of coronary atherosclerotic plaque by use of optical coherence tomography. Circulation, 2005; 111: 1551-1555.

4. Windecker S, Kolh P, Alfonso F et al. 2014 ESC/EACTS Guidelines on myocardial revascularization: The Task Force on Myocardial Revascularization of the European Society of Cardiology (ESC) and the European Association for Cardio-Thoracic Surgery (EACTS) Developed with the special contribution of the European Association of Percutaneous Cardiovascular Interventions (EAPCI). EuroIntervention 2014; 175: 240-247.

5. Prati F, Regar E, Mintz GS et al.; for the Expert's OCT Review Document. Expert review document on methodology, terminology, and clinical applications of optical coherence tomography: physical principles, methodology of image acquisition, and clinical application for assessment of coronary arteries and atherosclerosis. Eur Heart J, 2010; 31: 401-415.

6. Virmani R, Burke AP, Farb A, Kolodgie FD. Pathology of the vulnerable plaque. J Am Coll Cardiol, 2006; 47 (suppl. C): C13-C18.

7. Kubo T, Imanishi T, Takarada S et al. Assessment of culprit lesion morphology in acute myocardial infarction. J Am Coll Cardiol, 2007; 50: 933-939.

8. Jia $\mathrm{H}$, Abtahian $\mathrm{F}$, Aguirre $\mathrm{AD}$ et al. In vivo diagnosis of plaque erosion and calcified nodule in patients with acute coronary syndrome by intravascular optical coherence tomography. J Am Coll Cardiol, 2013; 62: 1748-1758.

9. Escaned J, Echavarria-Pinto M, Gorgadze T et al. Safety of lone thrombus aspiration without concomitant coronary stenting in selected patients with acute myocardial infarction. EuroIntervention, 2013; 8: 1149-1156.

10. Sianos G, Papafaklis MI, Daemen J et al. Angiographic stent thrombosis after routine use of drug-eluting stents in ST-segment elevation myocardial infarction: the importance of thrombus burden. J Am Coll Cardiol, 2007; 50: 573-583.

11. Onuma Y, Thuesen L, van Geuns RJ et al.; TROFI Investigators. Randomized study to assess the effect of thrombus aspiration on flow area in patients with ST-elevation myocardial infarction: an optical frequency domain imaging study: TROFI trial. Eur Heart J, 2013; 34: 1050-1060.

12. Svilaas T, Vlaar PJ, van der Horst IC et al. Thrombus aspiration during primary percutaneous coronary intervention. N Engl J Med, 2008; 358: 557-567.

13. Imola F, Mallus MT, Ramazzotti V et al. Manual thrombus aspiration in acute coronary syndrome: frequency-domain optical coherence tomography efficacy evaluation. Eur Heart J, 2011; 32 (abstract suppl.): 401.

14. Di Giorgio A, Capodanno D, Ramazzotti V et al. Optical coherence tomography guided in-stent thrombus removal in patients with acute coronary syndromes. Int J Cardiovasc Imaging, 2013; 29: 989-996.

15. Mintz GS, Painter JA, Pichard AD et al. Atherosclerosis in angiographically normal coronary artery reference segments: An intravascular ultrasound study with clinical correlations. J Am Coll Cardiol, 1995; 25: 1479-1485.

16. Costa MA, Angiolillo DJ, Tannenbaum Met al.; STLLR Investigators. Impact of stent deployment procedural factors on long-term effectiveness and safety of sirolimus-eluting stents (final results of the multicenter prospective STLLR trial). Am J Cardiol, 2008; 101: 1704-1711.

17. Legutko J, Jakala J, Mintz GS et al. Radiofrequency-intravascular ultrasound assessment of lesion coverage after angiography-guided emergent percutaneous coronary intervention in patients with non-ST elevation myocardial infarction. Am J Cardiol, 2013; 112: 1854-1859.

18. Legutko J, Jakala J, Mintz GS et al. Virtual histology-intravascular ultrasound assessment of lesion coverage after angiographically-guided stent implantation in patients with ST elevation myocardial infarction undergoing primary percutaneous intervention. Am J Cardiol, 2012; 109: 1405-1410.

19. Tanaka A, Imanishi T, Kitabata $\mathrm{H}$ et al. Lipid-rich plaque and myocardial perfusion after successful stenting in patients with non-ST segment elevation acute coronary syndrome: an optical coherence tomography study. Eur Heart J, 2009; 30: 1348-1355.

20. Prati F, Guagliumi G, Mintz GS et al. Expert Review Document part 2: methodology, terminology and clinical applications of optical coherence tomography for the assessment of interventional procedures. Eur Heart J, 2012; 33: 2513-2522.

21. Prati F, Di Vito L, Biondi-Zoccai G et al. Angiography alone versus angiography plus optical coherence tomography to guide decision-making during percutaneous coronary intervention: the Centro per la Lotta contro l'Infarto-Optimisation of Percutaneous Coronary Intervention (CLI-OPCI) study. EuroIntervention, 2012; 8: 823-829.

22. Gonzalo N, Serruys PW, Okamura Z et al. Optical coherence tomography assessment of the acute effects of stent implantation on the vessel wall: a systematic quantitative approach. Heart, 2009; 95: 1913-1919.

23. Gutierrez-Chico JL, Wykrzykowska J, Nuesch E et al. Vascular tissue teaction to acute malapposition in human coronary arteries. Sequential assessment with optical coherence tomography. Circ Cardiovasc Interv, 2012; 5: 20-29.

24. Raeber L, Radu MD. Optimising cardiovascular outcomes using optical coherence tomography-guided percutaneous coronary interventions. EuroIntervention, 2012; 8: 765-771.

25. Mueller C, Hodgson JB, Schindler C et al. Cost-effectiveness of intracoronary ultrasound for percutaneous coronary interventions. Am J Cardiol, 2003; 91: 143-147. 